\title{
Desempenho de crianças nascidas pretermo e em idade escolar na escrita: revisáo da literatura
}

\author{
Julie Souza de Medeiros Rocha ${ }^{a}$, Lilian de Fátima Dornelas ${ }^{b}$, Cynthia Girundi da Silva ${ }^{c}$, \\ Adriana Maria Valladáo Novais Van Petten ${ }^{\mathrm{d}}$, Lívia de Castro Magalhães ${ }^{\mathrm{d}}$
}

aSecretaria de Estado de Saúde do Distrito Federal, Especialista em Terapia Ocupacional no Desenvolvimento Infantil, Brasília, DF, Brasil

'Prefeitura Municipal de Uberlândia, Uberlândia, MG, Brasil

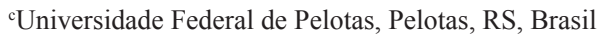

dDepartamento de Terapia Ocupacional, Escola de Educação Física, Fisioterapia e Terapia

Ocupacional - EEFFTO, Universidade Federal de Minas Gerais - UFMG, Belo Horizonte, MG, Brasil

\begin{abstract}
Resumo: Introdução: Na idade escolar a escrita é uma das tarefas mais relevantes dentre as atividades motoras finas. Bom desempenho na escrita contribui para o rendimento escolar da criança. A prematuridade tem impacto no desenvolvimento motor, o que pode influenciar a escrita. Objetivo: Revisar a literatura e investigar se na idade escolar existem diferenças no desempenho na escrita entre crianças nascidas pretermo e a termo. Método: Foram realizadas buscas nas bases de dados eletrônicas do Portal da Capes, nos idiomas inglês e português, no período de janeiro de 2000 a junho de 2012. Os artigos foram selecionados de acordo com critérios de inclusão e analisados quanto à amostra, objetivos, testes utilizados e desfechos. Resultados: Seis artigos foram incluídos no estudo. Análise dos dados indica que crianças nascidas prematuramente apresentam pior desempenho da escrita e prejuízo nos mecanismos subjacentes responsáveis pela habilidade de escrever quando comparadas com crianças a termo. Conclusão: Crianças pretermo e sem alterações neurológicas evidentes têm risco aumentado para prejuízo no desenvolvimento da escrita na idade escolar. O número reduzido de estudos recentes encontrados sobre este tema indica a necessidade de novas pesquisas, além do desenvolvimento de recursos padronizados para avaliação motora e da escrita em crianças brasileiras.
\end{abstract}

Palavras-chaves: Prematuridade, Pretermo, Escrita Manual, Destreza Motora.

\section{Handwriting performance of preterm children at school age: a literature review}

\begin{abstract}
Introduction: At school age, handwriting is on of the most important tasks among the fine motor activities. Good handwriting performance contributes to the child's school performance. As prematurity impacts on motor development, it may influence handwriting. Objective: To review the specific literature and investigate whether there are differences in handwriting performance at school age between children born preterm and full term. Method: A search was performed in the Capes electronic database, in English and Portuguese, comprising the time period between January 2000 and June 2012. Articles were selected according to the inclusion criteria; the sample, objectives, instruments utilized and outcomes were analyzed. Results: Six articles were included in the study. Data analysis indicates that children born prematurely present poorer handwriting performance and a handicap in the underlying handwriting mechanisms when compared with full term infants. Conclusion: Preterm children without obvious neurological impairment are at increased risk for problems in handwriting development at school age. The small number of recent studies on this topic indicates a need for further research, as well as the development of standardized resources for the motor and handwriting assessment of Brazilian children.
\end{abstract}

Keywords: Prematurity, Preterm, Handwriting, Motor Dexterity.

Autor para correspondência: Lívia de Castro Magalhães, Escola de Educação Física, Fisioterapia e Terapia Ocupacional, Universidade Federal de Minas Gerais, Av. Antônio Carlos, 6627, Campus Pampulha, CEP 31270-901, Belo Horizonte, MG, Brasil, e-mail: liviacmag@ gmail.com Recebido em Fev. 13, 2013; Revisão em Jul. 04, 2013; Aceito em Jul. 15, 2013. 


\section{Introdução}

A escrita é uma habilidade-chave no processo de aprendizagem escolar da criança e uma importante ferramenta de comunicação gráfica (FEDER; MAJNEMER, 2007). Na idade escolar, a escrita é a tarefa dominante dentre as atividades motoras finas, que consomem cerca de 30 a $60 \%$ do tempo gasto em sala de aula (MCHALE; CERMAK, 1992; MAGALHÁES et al., 2011a). O bom desempenho na escrita contribui tanto para o desenvolvimento acadêmico, social e da comunicação como para a construção da autoestima da criança (CORNHILL; CASE-SMITH, 1996).

Dificuldades na escrita são comumente observadas em crianças do ensino infantil. Segundo Sandler et al. (1992), entre 10 e 30\% das crianças apresentam dificuldades nesse aspecto sendo comumente caracterizadas como lentas ou desmotivadas. Os mecanismos subjacentes responsáveis pelas dificuldades na escrita ainda são pouco compreendidos, pois é uma atividade complexa, influenciada por fatores cognitivos, sociais, culturais e pelo próprio contexto de ensino-aprendizagem. Embora a alfabetização e ensino da escrita façam parte das competências dos professores, profissionais da reabilitação podem contribuir para essa área abordando a habilidade de escrita sob o ponto de vista de desempenho, pois geralmente este é o foco dos procedimentos de intervenção. Nesse contexto, vários estudos (FEDER; MAJNEMER, 2007; GOYEN; LUI; HUMMELL, 2011; MCHALE; CERMAK, 1992; VOLMAN; VAN SCHENDEL; JONGMANS, 2006) abordam componentes sensoriomotores, tais como o controle motor fino, a integração bilateral e visomotora, o planejamento motor, a manipulação manual, a propriocepçáo, a estereognosia, a percepção visual e a atenção, que dão suporte à escrita fluente e coordenada, necessária para o processo de aprendizagem e o bom desempenho escolar.

A prematuridade é um importante fator de risco para problemas no desenvolvimento, sendo que os déficits motores, perceptivo-motores e na integração visomotora, já bem documentados em crianças nascidas pretermo, podem limitar a competência na escrita (GOYEN; LUI; WOODS, 1998; LUOMA; HERRGARD; MARTIKAINEN, 1998; RUGOLO, 2005; VAN BRAECKEL et al., 2010; GOYEN; LUI; HUMMELL, 2011; SCHEPERS et al., 2012). Embora o peso ao nascer e a idade gestacional sejam fatores relevantes, devem ser considerados em combinaçáo com outros fatores de risco biológico, especialmente os de origem genética e as condiçóes provenientes de eventos danosos pré, peri ou pós-natais. Além disso, os fatores ambientais, associados a condiçóes precárias de saúde, à falta de recursos sociais e educacionais, aos estressores familiares e práticas inadequadas de cuidado pessoal, também devem ser considerados (GRAMINHA; MARTINS, 1997; HALPERN et al., 1996; MOURA et al., 2010).

No Brasil já existem vários programas de acompanhamento de crianças nascidas pretermo e é crescente o número de estudos brasileiros que documentam o atraso em diferentes áreas do desenvolvimento nessas crianças (CARDOSO; MAGALHÃES; BARBOSA, 2011; MAGALHÃES et al., 2003, 2009, 2011b; MOURA et al., 2010), no entanto, não há trabalhos que abordem especificamente a questão da escrita. Investigar o desempenho na escrita dessas crianças é fundamental, pois a identificação precoce de sinais relacionados, por exemplo, ao manejo do lápis, à velocidade na escrita, à formação de letras e caligrafia, pode possibilitar o encaminhamento precoce para a intervenção, minimizando problemas na idade escolar e vida adulta. Considerando a relevância da escrita na fase escolar e os possíveis problemas que crianças nascidas pretermo podem vir a apresentar, o objetivo deste estudo foi localizar e revisar os trabalhos publicados nacional e internacionalmente relacionados à habilidade na escrita em crianças nascidas prematuramente e investigar se há evidência de diferenças no desempenho entre crianças nascidas pretermo e a termo.

\section{Método}

Foi realizada busca bibliográfica nas bases de dados eletrônicas Medline, Lilacs, Pubmed, Scielo e Isi Web of Knowledge. As palavras-chave utilizadas foram: escrita, prematuridade, coordenação visomotora, handwriting, motor skill(s), motor performance, handwriting legibility, perceptual motor coordination, perceptual motor processes, hands skill, visual-motor ou visuo-motor, associadas aos termos premature e preterm. Para seleção dos artigos, foram usados os seguintes critérios de inclusão: trabalhos que incluíram avaliação do desempenho na escrita, publicados em inglês e português, no período de janeiro de 2000 a junho de 2012, cuja amostra contemplasse crianças nascidas abaixo de 37 semanas de gestação, sem alteraçôes neurológicas graves e com idade entre 5 e 8 anos. Os critérios de exclusão foram: estudos de caso e revisôes 
descritivas; artigos de avaliação das qualidades psicométricas de instrumentos relacionados ao desempenho na escrita.

Feitas as buscas, inicialmente os títulos dos trabalhos localizados foram analisados, descartando aqueles não pertinentes. Na sequência, foram lidos os resumos, sendo mantidos os artigos específicos de escrita. A sessão de metodologia desses artigos foi lida, sendo aplicados os critérios de inclusão, até se chegar aos trabalhos finais, específicos sobre o tema em estudo. Esses trabalhos foram lidos na íntegra, com análise detalhada da metodologia e dos resultados, de forma a construir a presente revisão.

A busca priorizou, inicialmente, apenas estudos publicados sobre o desempenho da escrita em crianças na idade escolar, nascidas prematuramente, mas como foi encontrado apenas um estudo (FEDER et al., 2005) abordando especificamente o desempenho funcional na escrita, a busca foi ampliada para incluir habilidades relacionadas à escrita, com uso de papel e lápis, tais como traçado e desenho. Assim, seis artigos foram incluídos para a análise crítica no presente estudo, como ilustrado na Figura 1.

\section{Resultados}

Para facilitar a apreciação das informações obtidas nos diferentes estudos, os dados foram resumidos e apresentados na Tabela 1 , no qual há

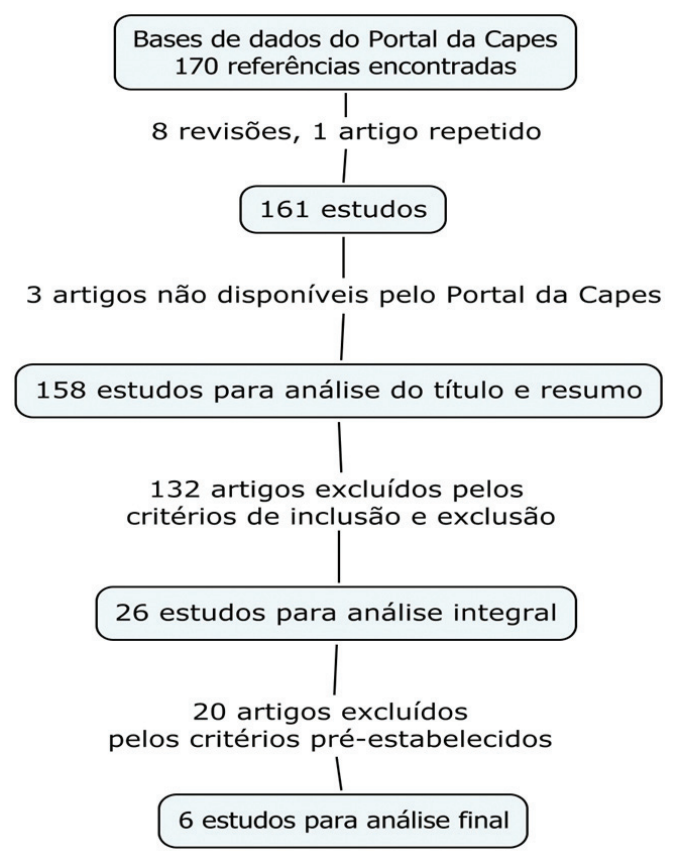

Figura 1. Fases do processo da revisão bibliográfica. descrição dos objetivos de cada estudo, participantes, testes utilizados e resultados encontrados.

Nos seis estudos analisados foram utilizados 22 instrumentos, sendo que apenas um (Evaluation Tool of Children's HandwritingManuscrip - ETCH-M) é específico para avaliação da escrita. Treze testes foram utilizados para avaliar mecanismos subjacentes relacionados à habilidade na escrita e os oito restantes para avaliar a cogniçáo e o comportamento, além do uso de análise eletromiográfica da ativação da musculatura envolvida na escrita (FLAMAND; NADEAU; SCHNEIDER, 2012). Foi observada predominância do uso dos testes Motor Accuracy (MAC) (AYRES, 1980), do Movement Assessment Battery for Children (M-ABC) (HENDERSON; SUGDEN, 1992), do Teste do Desenho da Figura Humana (AYRES; REID, 1966; WECHSLER, 1996), do Visual-Motor Integration (VMI) (BERRY, 1997) e do teste de Bender (KOPPITZ, 1975). Os instrumentos utilizados para avaliar a escrita, traçado e desenho estão descritos na Tabela 2.

\section{Discussão}

$\mathrm{Na}$ busca realizada foi encontrado apenas um estudo (FEDER et al., 2005) que avaliou a tarefa da escrita, propriamente dita, sendo que o restante dos estudos localizados (CARVALHO; MAGALHÃES, 2004; BOHM; LUNDEQUIST; SMEDLER, 2010; GOYEN; LUI; HUMMELL, 2011; FLAMAND; NADEAU; SCHNEIDER, 2012; SCHEPERS et al., 2012) focou a investigação nos componentes sensoriomotores da escrita e usou instrumentos de avaliaçáo que abordam aspectos ligados indiretamente à escrita, como provas de traçado e tarefas de coordenação visomotora.

Diante dos dados apresentados nos estudos, pode-se verificar que crianças nascidas pretermo, quando comparadas com crianças a termo, apresentam tanto desvantagens no desempenho funcional como déficits nos componentes subjacentes da escrita. Os artigos destacam que crianças pretermo e sem alteraçóes neurológicas evidentes apresentam, na idade escolar, lentidão e redução da acuidade/precisão na escrita, além de dificuldades na coordenação visomotora, visoespacial, percepção visual, coordenação motora fina e destreza manual (CARVALHO; MAGALHÁES, 2004; FEDER et al., 2005; BOHM; LUNDEQUIST; SMEDLER, 2010; GOYEN; LUI; HUMMELL, 2011; FLAMAND; NADEAU; SCHNEIDER, 2012; SCHEPERS et al., 2012). Os estudos 


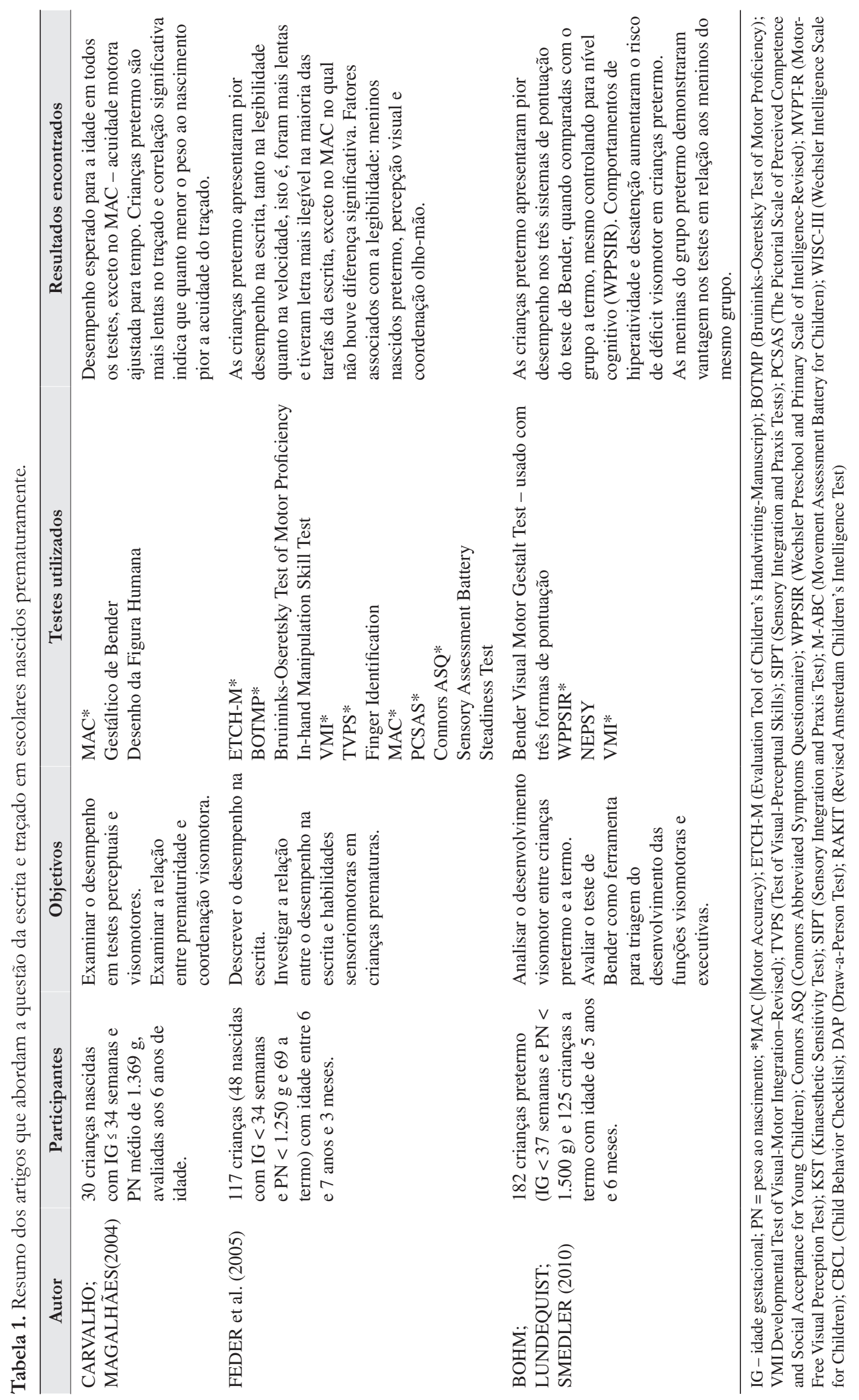




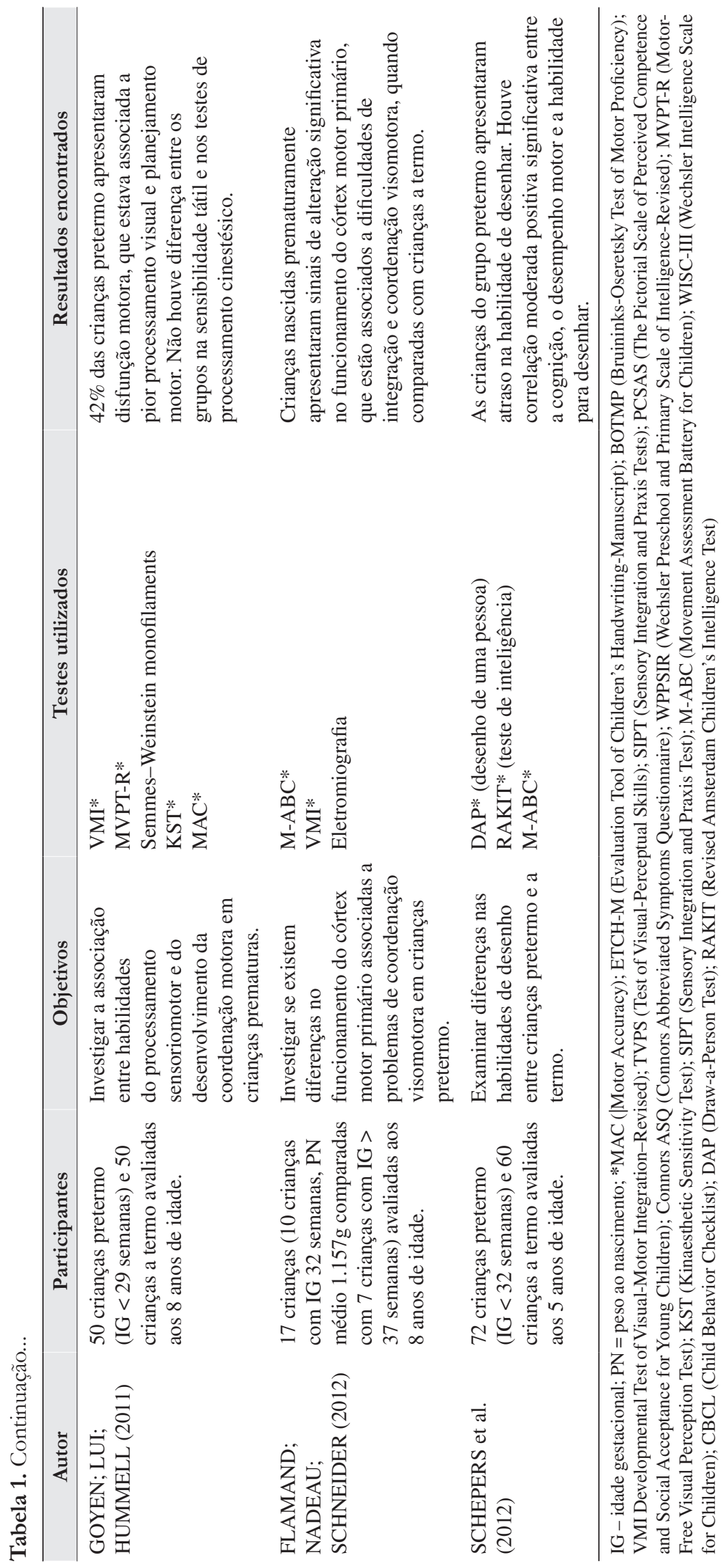




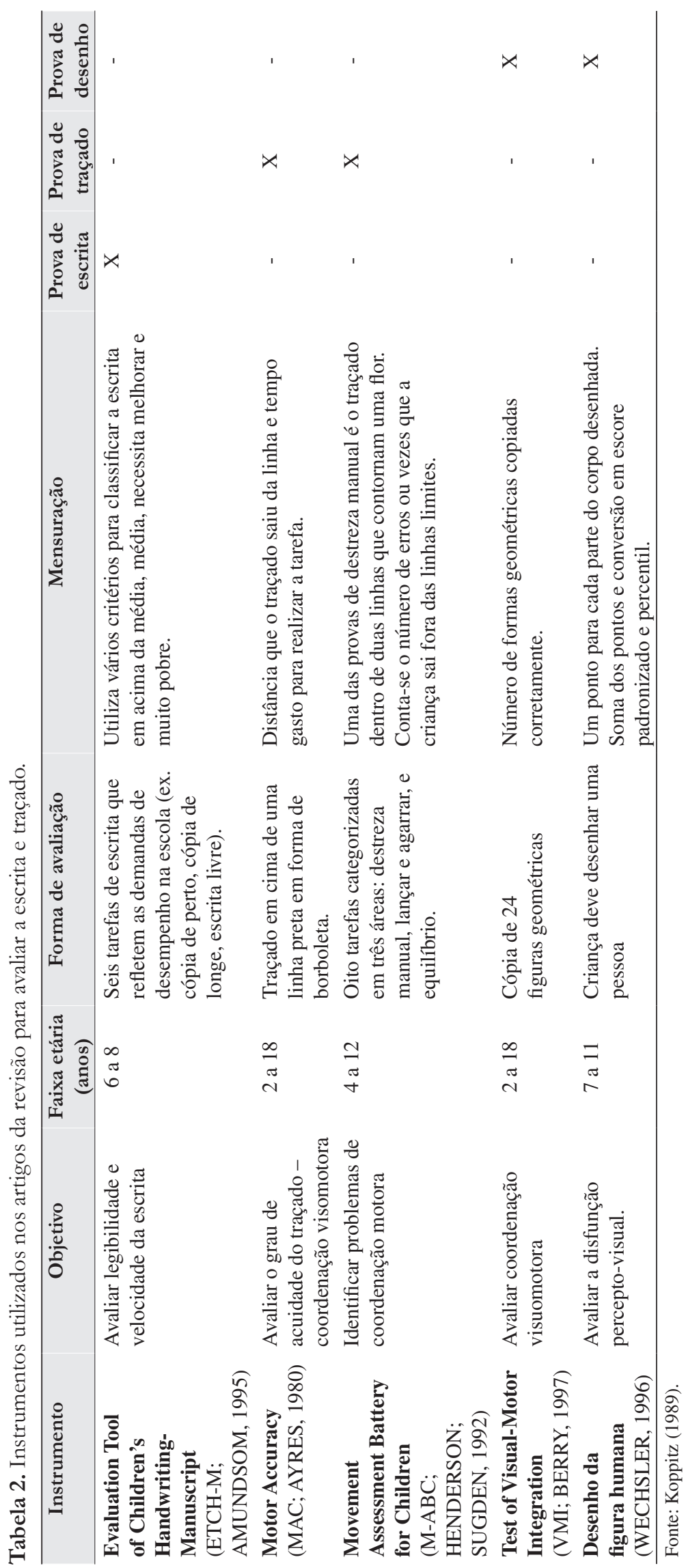




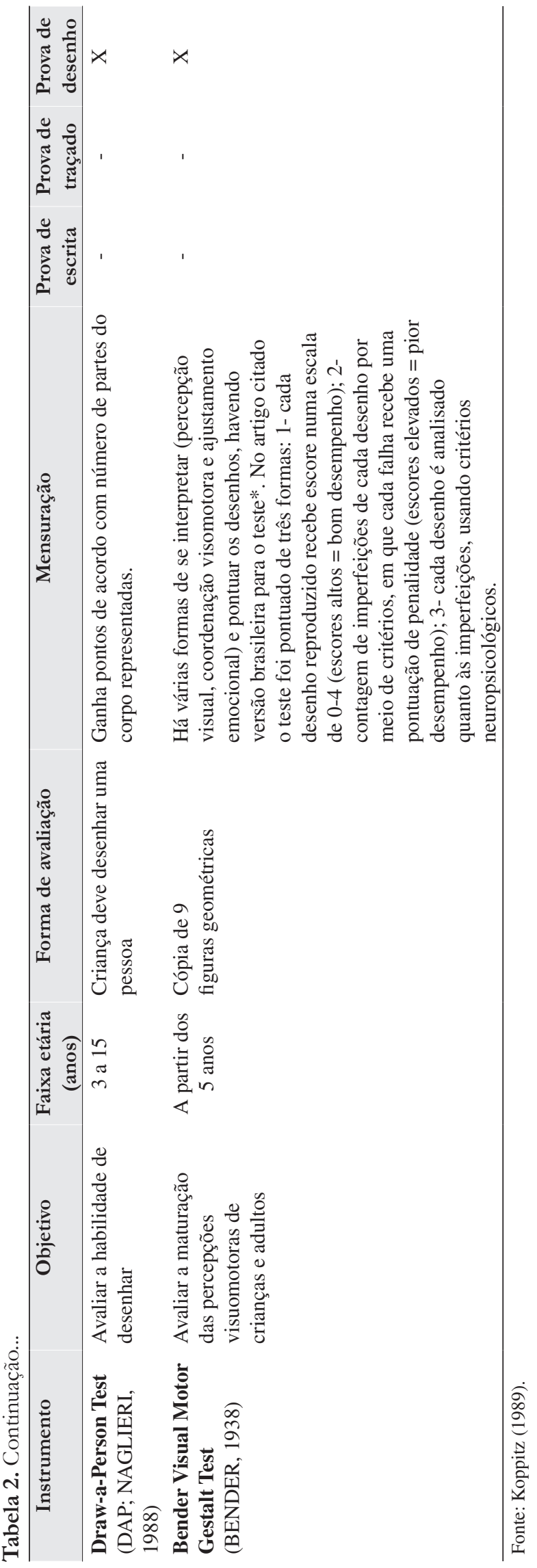


abordam vários componentes e não há consenso na literatura sobre quais deles seriam mais relevantes para a escrita.

Dos seis artigos selecionados, dois indicaram que as crianças pretermo apresentam lentidão na escrita e/ou provas de traçado (FEDER et al., 2005; CARVALHO; MAGALHÃES, 2004). Segundo estudo de Feder et al. (2005), a velocidade da escrita tem relação com as habilidades perceptivas e visuais da criança, e a maior lentidão nas crianças pretermo provavelmente está associada com a coordenação olho-mão, pois há evidência que esta habilidade nesse grupo é inferior, quando comparada com crianças nascidas a termo (GOYEN; LUI; HUMMELL, 2011).

Com relação à acuidade/precisão da escrita, os estudos indicaram que crianças pretermo apresentam pior legibilidade (FEDER et al., 2005), menor habilidade para desenhar (BOHM; LUNDEQUIST; SMEDLER, 2010; SCHEPERS et al., 2012) e menor acuidade/precisão no traçado (CARVALHO; MAGALHĀES, 2004; GOYEN; LUI; HUMMELL, 2011). Segundo Schepers et al. (2012), a habilidade para desenhar tem relaçáo com o desenvolvimento motor e cognitivo da criança, sendo que na criança pretermo a pior legibilidade possivelmente está associada a combinação de fatores motores e cognitivos, pois há evidência de que o desempenho cognitivo de crianças nascidas pretermo é ligeiramente inferior ao de pares nascidos a termo (RUGOLO, 2005; BOHM; LUNDEQUIST; SMEDLER, 2010). Alguns autores (FEDER et al., 2005; BOHM; LUNDEQUIST; SMEDLER, 2010) ressaltam, ainda, que distúrbios de comportamento comuns na criança pretermo, como a hiperatividade e déficit de atenção, são fatores de "confusão" na análise da legibilidade e velocidade da escrita nesse grupo de crianças.

Com relação a fatores individuais que podem interferir nessa tarefa, nos estudos de Feder et al. (2005) e Schepers et al. (2012), meninos prematuros tiveram pior desempenho na escrita. Esse dado é consistente com a literatura, pois em crianças nascidas a termo, a legibilidade da escrita das meninas é significativamente melhor que a dos meninos (ZIVIANI; WATSON-WILL, 1998). As condiçóes perinatais também parecem influenciar o processo de escrita, pois foi encontrada associação indicativa de que quanto menor o peso e a idade gestacional ao nascer pior a habilidade na escrita e/ou acuidade do traçado (CARVALHO; MAGALHÃES, 2004; FEDER et al., 2005). A presença de sinais de transtorno do desenvolvimento da coordenação, mais frequente entre crianças nascidas pretermo, também contribui para pior desempenho em provas de traçado (GOYEN; LUI; HUMMELL, 2011).

Deve-se ressaltar que o desempenho na escrita em crianças nascidas a termo foi investigado por vários autores (CORNHILL; CASE-SMITH, 1996; FEDER; MAJNEMER, 2007; MACHADO; CAPELLINI, 2011; ROSENBLUM; DVORKIN; WEISS, 2006; SANDLER et al., 1992; PEREIRA; ARAÚJO; BRACCIALLI, 2011; VOLMAN; VAN SCHENDEL; JONGMANS, 2006), entretanto, em crianças nascidas pretermo, essa habilidade ainda é menos pesquisada. Além de existirem poucos estudos recentes sobre a avaliação de desempenho na escrita em escolares nascidos prematuramente, apenas um dos estudos utilizou instrumentação específica para avaliar essa habilidade (FEDER et al., 2005). A maioria dos testes de papel e lápis usados nos estudos revisados (MAC, M-ABC, Desenho da Figura Humana e VMI) aborda aspectos relacionados apenas de maneira indireta à escrita (Tabela 2).

O Motor Accuracy Test (MAC) (AYRES, 1980) avalia a coordenação visomotora, examinando a acuidade com que a criança traça em cima de uma linha em formato de borboleta, que circunda a folha de teste. Faz-se a medida da distância que o traçado saiu da linha e do tempo gasto na tarefa. Embora sejam dados dois escores, um para acuidade e outro para a combinação entre o tempo gasto na prova e a distância traçada fora da linha, o teste não inclui escrita.

O Movement Assessment Battery for Children - M-ABC (HENDERSON; SUGDEN, 1992) é usado para identificar problemas de coordenação motora, avaliando a habilidade por meio de oito tarefas, divididas em três áreas: três itens de habilidades com bola, dois itens de destreza manual, sendo um deles uma prova de traçado (trilha da flor), e três de equilíbrio estático e dinâmico. Em 2007, foi lançada a segunda edição do teste (M-ABC II) que ampliou a faixa etária do teste para 3 a 14 anos, com itens similares, mas também não inclui avaliação específica da escrita (HENDERSON; SUGDEN; BARNETT, 2007).

O teste do Desenho da figura humana foi usado nos estudos revisados para avaliar a coordenaçáo visomotora e o esquema corporal, sendo usadas diferentes versóes do teste. $\mathrm{Na}$ versão de Wechsler (1996) a criança ganha um ponto para cada parte do corpo representada no desenho, de acordo com critérios específicos para correção do teste. O resultado bruto é obtido pela soma dos pontos 
e pode ser convertido em percentil e escore padronizado, o que permite estimar o nível de desempenho da criança em relação à sua faixa etária. Na versão de Ayres e Reid (1966), a pontuação é dividida em duas partes, na primeira, a criança perde pontos para cada parte do corpo humano não desenhada e na segunda, ela ganha pontos para cada detalhe encontrado no desenho. O escore final é dado pela soma dos pontos, sendo que, quanto maior a pontuaçáo positiva e a pontuação final convertida, melhor o desempenho da criança. Schepers et al. (2012) usaram uma versão cognitiva do teste, na qual as características das partes do corpo representadas são avaliadas em escalas de 0 a 7 pontos, com base na presença, nível e detalhe e proporcionalidade.

O Visual-Motor Integration (VMI) (BERRY, 1997), para avaliação da coordenação visomotora, consiste na cópia de 24 figuras geométricas com graus crescentes de dificuldade. A cada figura correta é dado um ponto e o somatório de pontos é convertido em escore padronizado para a idade. O escore do VMI tem boa correlação com a habilidade de escrita (DALY; KELLEY; KRAUSS, 2003). O teste gestáltico de Bender (KOPPITZ, 1975) tem similaridades com o VMI, pois se trata de cópia de nove desenhos geométricos, mas tem diferentes versôes, com critérios de pontuação e formas de interpretação específica.

Chama atenção o fato de que, embora existam diversos testes para avaliar especificamente a escrita (PHELPS; STEMPEL, 1987; LARSEN; HAMMIL, 1989; REISMAN, 1993; AMUNDSON, 1995), dentre os seis estudos revisados apenas o ETCH-M (AMUNDSON, 1995) foi utilizado em um único estudo. Esse achado pode ser justificado pela dificuldade de avaliar a escrita por meio de instrumentos padronizados. Como comentado por Feder, Majnemer e Synes (2000), profissionais de várias áreas, quando entrevistados, afirmaram ter dificuldade com a utilização de testes de escrita, preferindo utilizar testes motores globais a instrumentos específicos. Estudo sobre propriedades psicométricas de testes de escrita aponta que, apesar dos testes apresentarem bons índices de confiabilidade, há limitaçôes quanto ao uso na prática clínica devido ao complexo sistema de pontuação e escassez de estudos de validação (FEDER; MAJNEMER, 2003).

Cabe ressaltar que o uso de testes de escrita é apenas uma parte do processo de avaliação de Terapia Ocupacional, que deve também incluir a observação da criança em sala de aula e a opinião de pais e professores, assim como análise do lugar da escrita na vida ocupacional da criança. Utilizados dentro do devido contexto, esta revisão sugere que há necessidade de aperfeiçoar, desenvolver ou traduzir testes que possam ser úteis para avaliar a habilidade na escrita e auxiliar tanto na detecção de probemas como na avaliação de desfecho de programas de intervenção. Concluindo, há necessidade tanto de instrumentação como de estudos mais específicos sobre a qualidade da escrita e sobre a relação entre os componentes sensoriomotores e a escrita propriamente dita em crianças pretermo.

\section{Referências $^{1}$}

AMUNDSON, S. J. Evaluation Tool of Children's Handwriting. Homer, Alaska: OT Kids, 1995.

AYRES, A. J. Southern California sensory integration tests. Los Angeles: Western Psychological, 1980.

AYRES, A. J.; REID, W. The self-drawing as an expression of perceptual-motor dysfunction. Cortex, Amsterdam, v. 2, n. 2, p. 254-265, 1966. http://dx.doi. org/10.1016/S0010-9452(66)80007-2

BERRY, K. E. Revised administration, scoring, and teaching manual for the developmental test of visual-motor integration. Parsippany, New Jersey: Modern Curriculum Press, 1997.

*BOHM, B.; LUNDEQUIST, A.; SMEDLER, A. C. Visual-motor and executive functions in children born preterm: The Bender Visual Motor Gestalt Test revisited. Scandinavian Journal of Psychology, Malden, v. 51, p. 376-384, 2010. PMID: 20338020

CARDOSO, A. A.; MAGALHÃES, L. C.; BARBOSA, V. M. Desenvolvimento psicomotor em crianças pré-termo e a termo na idade escolar. Revista Brasileira de Crescimento e Desenvolvimento Humano, São Paulo, v. 21, n. 2, p. 210-219, 2011.

${ }^{*}$ CARVALHO, D. J.; MAGALHÂES, L. C. A relação entre o desenho da figura humana e a coordenaçáo visomotora em crianças pré-termo aos 6 anos de idade. Revista de Terapia Ocupacional da Universidade de São Paulo, São Paulo, v. 15, n. 3, p. 98-105, 2004.

CORNHILL, H.; CASE-SMITH J. Factors that relate to good and poor handwriting. American Journal of Occupational Therapy, Bethesda, v. 50, p. 732-739, 1996. http://dx.doi.org/10.5014/ajot.50.9.732

DALY, C. J.; KELLEY, G. T.; KRAUSS, A. Relationship between visual motor integration and handwriting skills of children in kindergarten: a modified replication study. American Journal Occupation Therapy, Bethesda, v. 57, p. 459-462, 2003. http://dx.doi.org/10.5014/ajot.57.4.459

*FEDER, K. P. et al. Handwriting performance in preterm children compared with term peers at age 6 to 7 years. Developmental Medicine and Child Neurology, Malden, v. 47, p. 163-170, 2005. http://dx.doi. org/10.1017/S0012162205000307 
FEDER, K. P.; MAJNEMER, A. Children's Handwriting Evaluation Tools and Their Psychometric Properties. Physical and Occupational Therapy in Pediatrics, Chicago, v. 23, n. 3, p. 65-84, 2003. http:// dx.doi.org/10.1080/J006v23n03_05

FEDER, K. P.; MAJNEMER, A. Handwriting development, competency, and intervention. Developmental Medicine and Child Neurology, Malden, v. 49, n. 4, p. 312-317, 2007. PMid:17376144. http:// dx.doi.org/10.1111/j.1469-8749.2007.00312.x

FEDER, K. P.; MAJNEMER, A.; SYNNES, A. Handwriting: Current trends in occupational therapy practice. Canadian Journal of Occupational Therapy, Switzerland, v. 67, n. 3, p. 197-204, 2000. http://dx.doi. org/10.1177/000841740006700313

*FLAMAND, V. H.; NADEAU, L.; SCHNEIDER, C. Brain motor excitability and visuomotor coordination in 8-year-old children born very preterm. Clinical Neurophysiology, Bethesda, v. 123, n. 6, p. 1191-1199, 2012. PMid:22018705. http://dx.doi.org/10.1016/j. clinph.2011.09.017

${ }^{*}$ GOYEN, T. A.; LUI, K; HUMMELL, K. L. Sensorimotor skills associated with motor dysfunction in children born extremely preterm. Early Human Development, Philadelphia, v. 87, n. 7, p. 489-493, 2011. PMid:21549531. http://dx.doi.org/10.1016/j. earlhumdev.2011.04.002

GOYEN, T. A.; LUI, K.; WOODS, R. Visual-motor, visual-perception and fine motor outcomes in very-lowbirth weight children at 5 years. Developmental Medicine and Child Neurology, Malden, v. 40, n. 2, p. 76-81, 1998. http://dx.doi.org/10.1111/j.1469-8749.1998.tb15365.x

GRAMinHA, S. S. V.; MARTins, M. A. Condiçóes adversas na vida de crianças com atraso no desenvolvimento. Medicina Ribeirão Preto, Ribeirão Preto, v. 30, n. 2, p. 259-267, 1997. http://dx.doi. org/10.11606/issn.2176-7262.v30i2p259-267

HALPERN, R. et al. Desenvolvimento neuropsicomotor aos 12 meses de idade em uma coorte de base populacional no Sul do Brasil: diferenciais conforme peso ao nascer e renda familiar. Cadernos de Saúde Pública, Rio de Janeiro, v. 12, n. 1, p. 73-78, 1996.

HENDERSON, S. E.; SUGDEN, D. A. Movement assessment battery for children. London: Psychological Corporation, 1992.

HENDERSON, S. E.; SUGDEN, D. A.; BARNETT, A. Movement Assessment Battery For Children-2 (MABC-2). San Antonio: The Psychological Corporation, 2007.

KOPPITZ, E. M. The Bender gestalt test for young children. Orlando: Grune \& Stratton, 1975. (Research and application, 1963-1973, v. 2).

KOPPITZ, E. O teste gestáltico de Bender para crianças. Porto Alegre: Artes Médicas, 1989.

LARSEN, S.; HAMMILL, D. Test oflegible Handwriting. Austin: PRO-ED, 1989.

LUOMA, L.; HERRGARD, E.; MARTIKAINEN, A. Neuropsychological analysis of the visuomotor problems in children born preterm at $<32$ weeks of gestation: a 5-year prospective follow-up. Developmental Medicine and Child Neurology, Malden, v. 40, n. 1, p. 21-30, 1998. http://dx.doi.org/10.1111/j.1469-8749.1998.tb15352.x

MACHADO, A. C.; CAPELLINI, S. A. Caracterização do desempenho de crianças com dislexia do desenvolvimento em tarefas de escrita. Revista Brasileira Crescimento e Desenvolvimento Humano, São Paulo, v. 21, n. 1, p. 132-138, 2011.

MAGALHÃES, L. C. et al. Relação entre destreza manual e legibilidade da escrita em crianças: estudo piloto. Revista de Terapia Ocupacional da Universidade de São Paulo, São Paulo, v. 22, n. 2, p. 127-135, 2011 a. MAGALHÁES, L. C. et al. Desempenho de crianças pré-termo com muito baixo peso e extremo baixo peso segundo o teste Denver-II. Revista Brasileira Saúde Materno Infantil, Recife, v. 11, n. 4, p. 445-453, 2011 b. http://dx.doi.org/10.1590/S1519-38292011000400011

MAGALHÁES, L. C. et al. Análise comparativa da coordenação motora de crianças nascidas a termo e pré-termo, aos 7 anos de idade Revista Brasileira Saúde Materno Infantil, Recife, v. 9, n. 3, p. 293-300, 2009.

MAGALHÁES, L. C. et al. Estudo comparativo sobre o desempenho perceptual e motor na idade escolar em crianças nascidas pré-temo e a termo. Arquivos NeuroPsiquiatria, São Paulo, v. 61, n. 2A, p. 250-255, 2003. http://dx.doi.org/10.1590/S0004-282X2003000200016

MCHALE, K.; CERMAK, S. A. Fine motor activities in elementary school: preliminary findings and provisional implications for children with fine motor problems. American Journal of Occupational Therapy, Bethesda, v. 46, p. 898-903, 1992. http://dx.doi.org/10.5014/ ajot.46.10.898

MOURA, D. R. et al. Natural history of suspected developmental delay between 12 and 24 months of age in the 2004 Pelotas birth cohort. Journal of Paediatrics and Child Health, Malden, v. 46, n. 6, p. 329-336, 2010. PMid:20412410. http://dx.doi.org/10.1 $111 /$ j.1440-1754.2010.01717.

NAGLIERI, J. A. Draw a person: a quantitative scoring system. San Antonio: Psychological Corporation, 1988. PEREIRA, D. M.; ARAÚJO, R. C. T.; BRACCIALLI, L. M. P. Habilidade de integração visuo-motora e o desempenho escolar. Revista Brasileira Crescimento Desenvolvimento Humano, São Paulo, v. 21, n. 3, p. 808-817, 2011.

PHELPS, J.; STEMPEL, L. Children's handwriting evaluation scale for manuscript writing. Dallas: Texas Scottish Rite Hospital for Crippled Children, 1987.

REISMAN, J. E. Development and reliability of the research version of the Minnesota Handwriting Test. Physical and Occupational Therapy in Pediatrics, Chicago, v. 13 , n. 2, p. 41-55, 1993. http://dx.doi.org/10.1080/ J006v13n02_03

ROSENBLUM, S.; DVORKIN, A. Y.; WEISS, P. L. Automatic segmentation as a tool for examining the handwriting process of children with dysgraphic and proficient handwriting. Human Moviment 
Scienc, Amsterdam, v. 25, n. 4, p. 608-621, 2006. PMid:17011656. http://dx.doi.org/10.1016/j. humov.2006.07.005

RUGOLO, L. M. S. S. Crescimento e desenvolvimento a longo prazo do prematuro extremo. Jornal de Pediatria, Porto Alegre, v. 81, p. S101-S110, 2005. Suplemento 1 http://dx.doi.org/10.2223/JPED.1309

SANDLER, A. D. et al. Neurodevelopmental study of writing disorders in middle childhood. Developmental and Behavioral Pediatrics, Baltimore, v. 13, n. 1, p. 17-23, 1992. PMID: 1556195

*SCHEPERS, S. et al. Drawings of very preterm-born children at 5 years of age: a first impression of cognitive and motor development? European Journal Pediatric, New York, v. 171, n. 1, p. 43-50, 2012. PMid:21547371 PMCid:PMC3249160. http://dx.doi.org/10.1007/ s00431-011-1476-8

VAN BRAECKEL, K. et al. Difference rather than delay in development of elementary visuomotor processes in children born preterm without cerebral palsy: a quasilongitudinal study. Neuropsychology, Washington, v. 24, n. 1, p. 90-100, 2010. PMid:20063950. http://dx.doi. org/10.1037/a0016804

VOLMAN, M. J. M.; VAN SCHENDEL, B. M.; JONGMANS, M. J. Handwriting difficulties in primary school children: a search for underlying mechanisms. American Journal Occupation Therapy, Bethesda, v. 60, n. 4, p. 451-460, 2006. http://dx.doi.org/10.5014/ ajot.60.4.451

WECHSLER, S. M. O desenho da figura humana: avaliação do desenvolvimento cognitivo infantil. Campinas: Psycho, 1996. Manual para crianças brasileiras.

ZIVIANI, J.; WATSON-WILL, A. Writing speed and legibility of 7-14-year-old school students using modern cursive script. Australian Occupational Therapy Journal, Malden, v. 45, n. 2, p. 59-64, 1998. http://dx.doi. org/10.1111/j.1440-1630.1998.tb00783.x

\section{Contribuição dos Autores}

Julie Souza de Medeiros Rocha: concepção do estudo, buscas e redação original do texto. Lílian de Fátima Dornelas: concepção, redaçáo e revisão do texto. Cynthia Girundi da Silva e Lima: revisão da literatura e do texto. Adriana Maria Valadão Novais Van Peten: revisão e finalização do texto. Lívia de Castro Magalhães: concepção do estudo, redação e revisão do texto. Todas as autoras aprovaram a versão final do texto.

\section{Notas}

${ }^{1}$ Os artigos revisados estão sinalizados com *. 